\title{
Review on Principles of Zoonoses Prevention, Control and Eradication
}

\author{
Haregua Teshome* and Shimeles Abegaz Addis \\ School of Veterinary Medicine, Wollo University, Ethiopia \\ *Corresponding author: Haregua Teshome, School of Veterinary Medicine, Wollo University, PO Box: 1145, Dessei Amhara, Ethiopia. \\ To Cite This Article: Haregua Teshome. Review on Principles of Zoonoses Prevention, Control and Eradication. Am J Biomed Sci \& Res. 2019 - \\ 3(2). AJBSR.MS.ID.000660. DOI: 10.34297/AJBSR.2019.03.000660
}

Received: May 07, 2019 | Published: June 06, 2019

\begin{abstract}
Zoonoses are "those diseases and infection which are naturally transmitted between vertebrate animals and man". The transmission may occur through direct contact with the animal, through vector (such as fleas or tick), or through food or water contamination. Zoonotic diseases cause mortality and morbidity in people, while also imposing significant economic losses in the livestock sector. Zoonosis constitutes a diverse group of viral, bacterial, rickettsia, fungal, parasitic and prion disease with a variety of animal reservoirs, including wildlife, livestock, pet animals and birds. The basic principles of zoonoses prevention control and eradication involves reservoir neutralization, reducing contact potential and increasing host resistance. Reservoir neutralization involves preventing spread of infection by removing the infected individual from the reservoir or by manipulation the environment where the reservoir resides. The removal of infected individual can be accomplished by means of a test and slaughter, and mass therapy.

Test and slaughter have been most effective with agents spread by direct transmission and in which a limited number of reservoir species are involved. Mass therapy is usually restricted to a local situation in which all potential infected animals or people are treated without first testing them to identify infected individuals. Environmental manipulation can be designed to decrease environmental contamination by acting in relation to the portal of exit from the infected hosts or by decreasing exposure of susceptible hosts by acting in relation to the portal of entry. A basic principle in preventing direct transmission of an infectious agent from an infected individual to a susceptible host is to reduce the opportunity for contact between the two. Increasing host resistance may prevent infection of susceptible hosts or reduce severity of disease. Host resistance may be increased by genetic selection, stress reduction, chemoprophylaxis or immunization. The link among humans, animal populations and the surrounding environment should be done with proper care in order to protect serious consequence both for humans and for economically animals. Health professionals should educate the public about zoonotic diseases to effectively practice prevention, control and eradication strategies.
\end{abstract}

Keywords: Control; Eradication; Prevention; Zoonoses, Chemoprophylaxis; Immunization

\section{Introduction}

The link among humans, animal population and the surrounding environment is very close in many developing countries, where animals provide transportation, draught power, fuel, clothing and source of protein (that is meat, eggs, and milk). In the absence of proper care, this linkage can lead to a serious risk to public health with huge economic consequences [1].

Zoonoses are defined as those diseases and infection naturally transmitted between people and vertebrate animals [2] zoonoses constitute a diverse group of viral, bacterial, rickettsial, fungal, parasitic and prion disease with a variety of animal reservoir, including wildlife, livestock, pet animals and birds [3]. The transmission may occur through direct contact with the animal, through vectors (such as fleas or tick), or through food or water contamination. Globally zoonoses are said to account for $60 \%$ of all infectious disease pathogens and 75\% of all emerging pathogens [4]. In both developing and developed countries, several new zoonoses have emerged. This might be the result of either newly discovered pathogens or agents that are already known, usually appearing in animal species in which the disease had not previously been detected [5]. Many diseases that affect human which are new, emerging and reemerging were caused by pathogens that originated from animal. Moreover, several zoonotic diseases including rabies, brucellosis, bovine tuberculosis and echinococcosis continue to affect human and animals in many countries, particularly developing nations [6].

It has been observed that $75 \%$ of emerging pathogens fall within the category of zoonotic disease. Zoonotic disease caused mortality and morbidity in people, while also imposing significant economic losses in the livestock sector. Their burden tends to 
fall most heavily on poor societies [2]. They have both direct and indirect effects on livestock health and production [7]. Indirect effects occur as a result of the risk of human disease, the economic impact on livestock producers through barriers to trade, the costs associated with control programmers, the increased cost of marketing produce to ensure it is safe for human consumption, and the loss of markets because of decreased consumer confidence [8].

In most developing countries, zoonotic disease is among those diseases that contribute significantly to an already overly burdened public health system. In industrialized nation zoonotic disease are of a concern for at risk group such as the elderly, children, childbearing women and immune-compromised individuals. Many different determinants contribute to the emergence of a new zoonotic agents and it is rare that this factor act singly. Among the forces that shape their emergence are human demographics and behaviors, technology, industry and agriculture, economic development and land use, international travel, commercial and military expedition, microbial adaptation and change and breakdown of public health measure. Indeed, social and environmental changes are accelerating in both the developed and developing worlds. The developed world has the greatest travel and transport, providing particular risk for rapid spread. Ecological change is greatest in the developing world and biodiversity is greatest in the tropics, which makes these region potentially productive breeding grounds for new pathogens [9].

To conclude, it cannot be predicted, which zoonotic pathogens are likely to emerge next or cause the biggest problem. Given the obvious link between human health and pathogens that circulate in domestic animals and wildlife, we must be alert to pathogen flow in any of these areas. Therefore, the objectives of this seminar paper are

1. To give highlight on the general zoonoses etiology, transmission and classification

2. To provide information about the socio-economic significance of zoonoses

3. To review principles of zoonoses prevention, control and eradication.

\section{Definition of Zoonosis}

Several definitions of a zoonoses are available, some of which are reported here below: "any disease and/or infection which is naturally transmissible directly or indirectly between animal and humans" [10]. A disease or infection which is naturally transmissible from animal to humans" [11]. "Any disease or infection that is naturally transmissible from vertebrate animals to humans and vice versa".

Zoonoses are defined as infectious diseases that can be transmitted naturally between human and wild or domestic animals. These diseases are particularly important in the context of emerging infectious disease of humans as the majority of these are of zoonotic origin. A comprehensive review by cleave land et al. 2001 identified 1,415 species of infectious organisms known to be pathogenic to human, including 217 virus and prions, 538 bacteria and rickettsia, 307 fungi, 66 protozoa and 287 helminths. Out of these $868(61 \%)$ were classified as zoonotic and 175 pathogenic species were associated with emerging disease. Of this group of 175 emerging pathogens 132 (75\%) were zoonotic. Whatever definition is considered, animals play an essential role in maintaining zoonotic infection in nature. Zoonoses may be bacterial, viral or parasitic or may involve prions [12]. As well as being a public health problem, many of the major zoonotic diseases prevent the efficient production of food of animal origin and create obstacles to international trade in animal products.

\section{Zoonotic disease transmission}

Transmission of infection requires three elements: a source of pathogens, a susceptible host and a means of transmission for the microorganism [13].

Source: Include animal or environment contaminated by animals. Pathogens may be transmitted to humans directly from the animal via blood or other body substances during diagnostic or treatment procedures or indirectly from the animal's environment [13] (Figure 1).

\section{Disease Ecology}

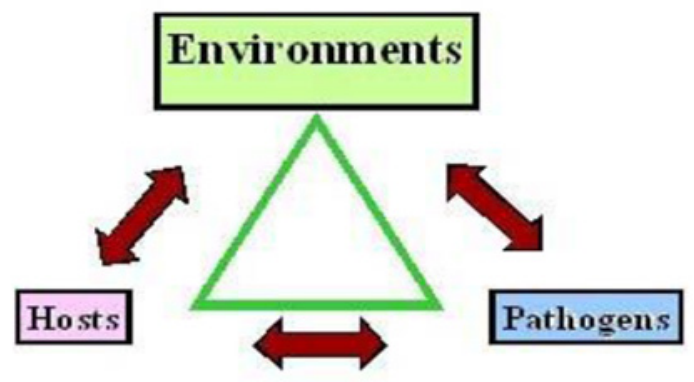

Figure 1: Zoonotic disease transmission.

Host Susceptibility: Animal may be clinically ill, asymptomatic carrier of an infectious agent, harbor endogenous flora that are pathogenic to human or in the incubation period of an infectious disease. Pathogens may also be transmitted indirectly from fomites in the environment including walls, floors, counters, equipment, supplies, animal feed and water. Host resistance to pathogenic microorganism varies greatly. Some people may be immune to infection or may be able to resist colonization by an infectious agent. Others exposed to the same agent may become asymptomatic carriers, while still others may develop clinical disease. Host factors such as age, underlying disease, immunosuppression, irradiation, pregnancy and breaks in the body's first line of defense mechanism (intact skin, cough, reflex, stomach acid) may render a host more susceptible to infection. Conversely vaccination may reduce susceptibility to infection [14].

Route of Transmission: Transmissions occur through three main mechanisms, direct or indirect contact, aerosol and vector borne. The same agent may be transmitted by more than one route. Transmission is largely influenced by the stability of the pathogen, its virulence and the route by which it leaves the infected host. Different agent varies in their degree of infectivity through the various route [14]. 
Contact Transmission: This can occur when pathogens from animals or their environments enter the human host through ingestion, mucous membrane or cutaneous/percutaneous exposure. Direct contact transmission may occur during activities such as examining, medicating, bathing and handling animals. Indirect contact transmission involves contact with a contaminated intermediate object (Fomites), occurs during cleaning cages and equipment's and handling soiled laundry. Injuries contaminated sharps such as scalpel blades, needles, and necropsy knives, may result in exposure to live vaccine and pathogens. In addition, injury from sharps increases risk of exposure to other pathogens through direct and indirect contact [13] (Figure 2).

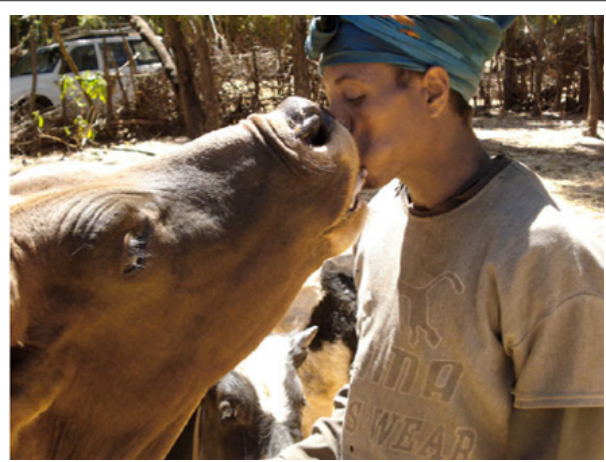

Figure 2: A farmer in central Ethiopia discharging tobacco juice directly into the oral cavity of his cattle. A common practice in this region and a possible route of transmission of mycobacterium tuberculosis from human to cattle (example of Zooanthroponoses).

Examples of zoonotic disease transmitted by direct contact and fomites include: Acariasis (mange), Anthrax, Avian Influenza, Brucellosis, Cat-scratch disease, Dermatophytosis, Glanders, Melioidosis, Monkeypox, Mycobacteriosis, Nipah, Pasteurellosis, Plague, Q-fever, Rabies, Ratbitefever,Sporotrichosis, streptococcosis, Tularemia etc. zoonotic disease that can be transmitted by ingestion are Avian influenza, Baylisascariasis, Campylobacteriosis, chlamidiosis (mammalian). Cryptosporidiosis, Escherichia coli 0157:H7, Echinococcosis, Giardiasis, Hookworm, Larva migrans, Salmonellosis, Toxoplasmosis, Tularemia, Yersiniosis etc.

Aerosol Transmission occurs when pathogens from animals or their environments travel via the air and enter the human host through inhalation and /or mucous membranes. In general, risk to veterinary personnel increases with proximity to the source and the length of time over which exposure occurs. Transmission over short distance occurs when droplet created by coughing, sneezing, vocalizing or procedures such as suctioning and bronchoscopy are propelled through the air and deposited on the hosts conjunctivae, nasal or oral mucosa [13].

Certain pathogens may remain infective over longer distances [14]. However, defining the infective distance is difficult because it depends on particle size, the nature of the pathogen, and environmental factor [15]. Although data are not available to define specific infection risk from rosol transmission for most pathogens, some pathogens known to be transmitted over longer distances include coxiella burnetii (Q-fever) [15-17] and mycobacterium bovis (bovine tuberculosis-not present in Australia since 1997)
[18]. Example of zoonotic disease transmitted through aerosol are Bordetella infection, Cryptococcosis, Hantavirus, Melioidosis, Nipah, plague, Psittacosis, Q-fever, streptococcosis, Tualremia etc. (www.cfsph.iastate.edu)

Vector-Borne Transmission: Vector such as mosquitos, feas, ticks, rats and other animals may transmit microorganisms. Animal may bring fleas and ticks into contact with veterinary personnel. Veterinary personnel working in outdoor settings may be at risk for disease carried by arthropods and other biological vectors. Example of zoonotic disease transmitted by an arthropod vectors: fleas-plague, mosquitoes- west Nile encephalitis, Ticks- ehrlichiosis, Leishmaniasis, lyme disease, q-fever, rocky mountain spotted fever, Tularemia, Triatomine ("kissing bugs") Trypanosomiasis (Chagas disease) (www.cfsph.iastate.edu) (Figure 3).

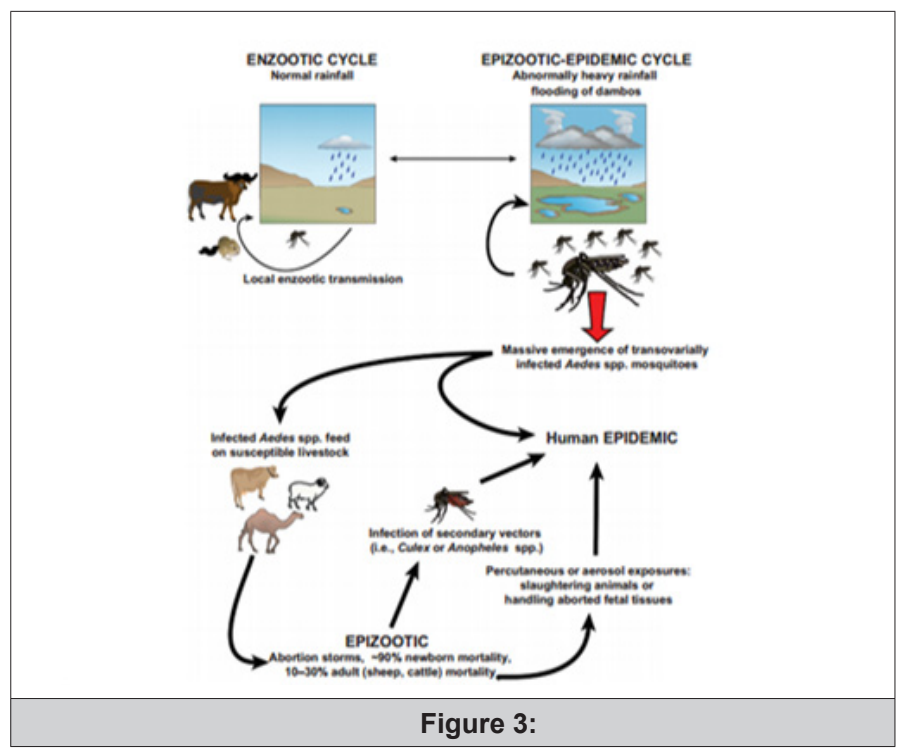

\section{Type of Zoonoses}

Reverse Zoonoses: Infectious disease of people occasionally transferred to animals and then transferred back to people is termed as "reverse zoonoses" [15]. Examples are Tuberculosis [16], Mumps, Streptococcus pyogenes, infectious hepatitis, coryne bacterium diphtheria [17].

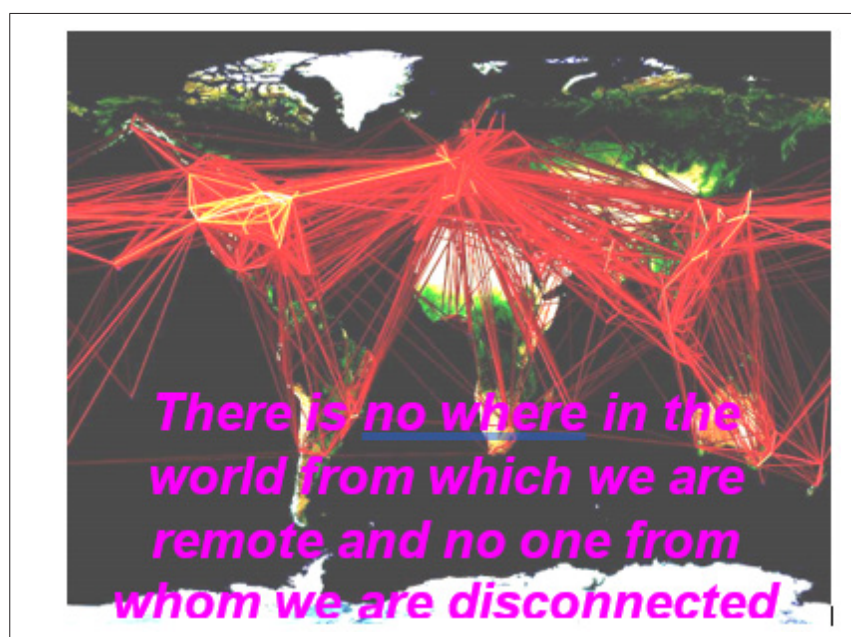

Figure 4: 
Emerging Zoonoses: Zoonosis that is newly recognized or newly evolved or that has occurred previously but shows an increase in incidence or expansion in geographical, host or vector range is known as emerging zoonosis. Country to "lingering" zoonoses, public awareness of "emerging" zoonoses is very high. Emerging zoonotic disease has potentially serious human health and economic impact and their current upward trends are likely to continue. The recent emerging zoonoses, for example are Bird flu, AIDS, Mad cow disease [18] and the Nipah virus [19]. Some of the "lingering" zoonoses are re-emerging in some regions, although they seem to attract less public awareness. These include brucellosis, dog rabies and parasitic disease such as cysticercosis/ taeniasis and echinococcosis/ hydatidosis [19] (Figure 4).

\section{Factors Influencing Emerging Zoonoses}

Many factors lead to the emergence of zoonotic disease. Environmental change, human and animal demography, pathogen changes and changes in farming practice are a few of them. Social and cultural factors such as food habits and religious beliefs play a role too. New animal disease with an unknown host spectrum is also included in this definition. Natural animal reservoir represents a more frequent source of new agent of human disease than the sudden appearance of a completely new agent [17].

Factors explaining the emergence of a zoonotic or potentially zoonotic disease are usually complex, involving mechanisms at the molecular level, such as genetic drift and shift, as it occurs in avian influenza virus [18] and modification of the immunological status of individuals and population. Social and ecological conditions influencing population growth and movement, food habits, the environment and many other factors may play a more important role than changes at the molecular level. Viruses especially RNA viruses with their ability to adopt quickly to changing environmental conditions are among the most prominent examples of emerging pathogens [17] (Figure 5).

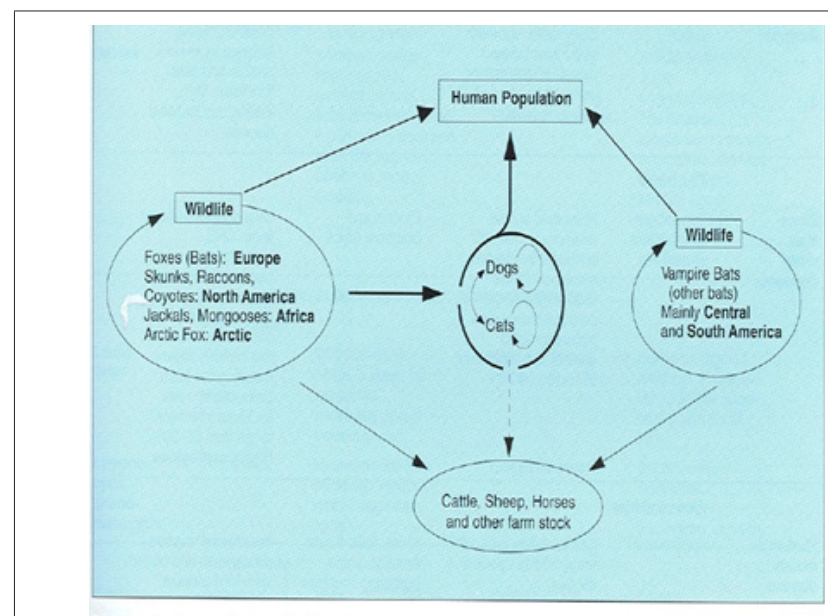

Diagram 68. Transmission of rabies from wildlife reservoirs to humans and to domestic animals.

Figure 5:

\section{Classification of Zoonoses}

Based on the type of life cycle of the Infective Organism: A classification system based on the type of life cycle of the infective organism seems the most useful in planning a preventive medicine program. The following categories are recommended by the World Health Organization Expert Committee of zoonoses [19].

Direct Zoonoses: Zoonoses transmitted from an infected vertebrate host to a susceptible vertebrate host by contact, vehicle or mechanical vector. Only a single vertebrate species is required to perpetuate this infection cycle and the agent itself under go little or no propagative chande and needs to undergo no essential developmental change during transmission. Most direct zoonosis is transmitted to man though direct physical contact with an animal. examples are rabies by the bite route, Dermatomycosis by touch contact, Anthrax tissue contact, Leptospirosis excretions (urine), influenza through indirect contact with air borne droplets, originating in respiratory tract of the animal, other direct zoonoses are vehicle borne, man becoming infected by the ingestion of contaminated raw meat-example Trichinosis, contaminated raw milk- example, Salmonellosis, Tuberculosis. Contaminated water example- Giardiasis, inhalation of dust example-Q-fever [20] (Figure 6).

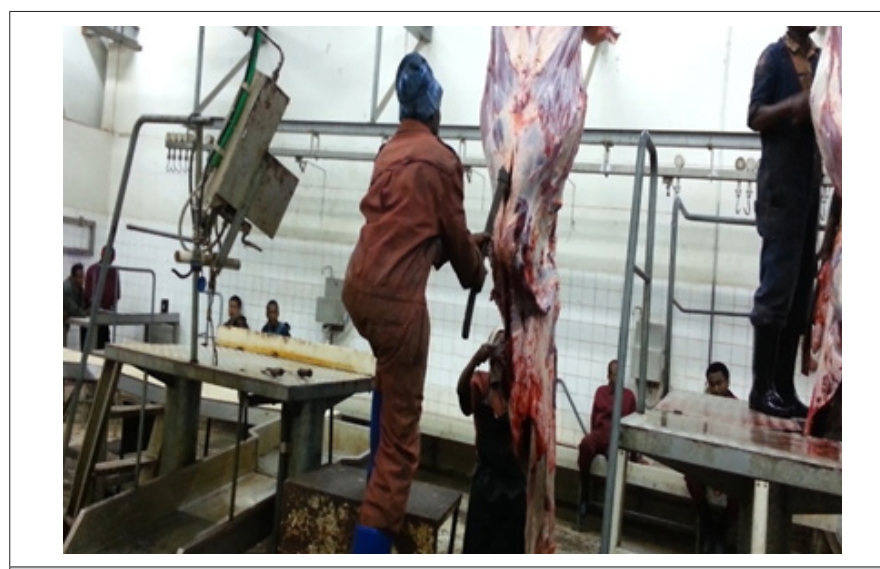

Figure 6: Breastbone splitting on a carcass using wood handled axe (unhygienic handling of edible carcass).

Cyclo-Zoonoses: Zoonoses whose maintenance cycle require more than one vertebrate host but no invertebrate host is required for the completion of the agent's developmental cycle. Obligatory cyclozoonoses: here man must be one of the vertebrate hosts in these cycles' examples: Taenia saginata and Taenia solium.

Non-Obligatory Cyclozoonoses: In such condition man is sometimes involved (an accidental host). example; Hydatidosis [20].

Meta Zoonoses: If the infection is transmitted biologically by the invertebrate vectors, the agent characteristically multiply or develop or do both in the invertebrate hosts and they essentially show a pre-patent period i.e., extrinsic incubation before being transmitted to a suitable vertebrate host), the zoonoses will be termed as a meta-zoonoses. Examples are arboviruses, Leishmaniasis, plague, and schistosomiasis [20].

Sapro Zoonoses: Zoonoses which require a non-animal site to serve either as a true reservoir of infection or as a site of for an essential phase of development. Non animal sites include organic 
matter including food, soil and plant. The agent may propagate in the non-animal site (type I) as in the case of histoplasmosis where soil enriched by bird or bat manure serves as a true reservoir. the agent may undergo essential development without propagation as in Ancylostoma brasiliense infection. Other example of saprozoonoses include botulism, Ascariasis, Trichuriasis, Visceral migrans e.g. toxocara canis, T cati) [2].

Depending upon the direction of transmission of zoonoses among man and lower vertebrates, the following classification exists (Figure 7).

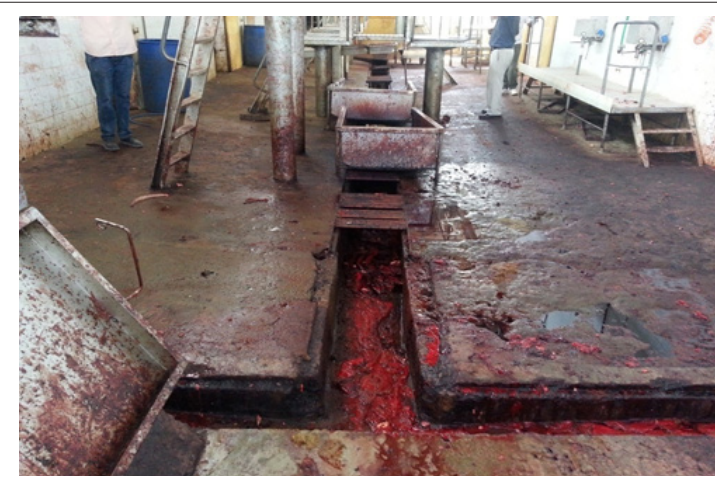

Figure 7: Poor drainage and sanitation, means of zoonoses transmission in the abattoir.

Anthropozoonosis: Infections transmitted to man from lower vertebrate. In this case the agents can all exist in mature independently of man. Example: rabies, brucellosis, hydatidosis etc., [1].

Zooanthroponoses: infection transmitted from man to animals. These are quite small groups of zoonotics diseases which normally pass from man to man, but which may infect other vertebrate animals. examples:n Tuberculosis of human type, Amoebiasis (entamoeba-histolytica only man, entamoeba-poleckiswine to man [2].

Amphixenoses: Infection maintained in both man and lower vertebrates. Humans and lower vertebrates are equally suitable reservoir hosts and infection may be transmitted in either direction. Examples; staphylococcoses, streptococcoses. Zoonotic diseases/ infections may also be classified according to the type of etiologic agents involved.

Viral Zoonoses: Are virus infections of animals that can be naturally transmitted to man, often with devastating effect. Rabies is perhaps the prime example of a zoonotic viral infection which causes some 60,000 human deaths per year. Rabies is a disease of carnivores and bats, which is mainly transmissible to human by bits. Almost all persons severely exposed to rabid animals die if not treated. An estimated number of 55,000 person, mainly children die of this disease in the world every year [17]. Other viral zoonoses are avian influenza, Crimean congo-haemorrhagic fever, Ebola and Rift valley fever [16].

Bacterial Zoonoses: every year millions of people get sick, because of food borne zoonoses caused by different types of pathogenic bacteria such as Salmonellosis, Campylobacteriosis, erysipelas, leptospirosis, listeriosis, tuberculosis, anthraz, Brucellosis, plague, Shigellosis, Tularaemia [19].

Rickettsial zoonoses: Rickettsiae are extremely small sized obligate intracellular prokaryotes, which multiply by binary fission. Rickettssial disease are primarily transmitted by arthropods. The major reservoirs of infection are humans, rats, mice and small ruminants [20]. The main source of human infection is affected domestic animals and their products. Human patients can develop a chronic illness characterized by endocarditis and hepatitis [21] examples of Rickettssial zoonosis includes Rocky Mountain spotted fever, Q-fever, avian chlamydiosis [16]

Parasitic Zoonoses: Parasitism is the major health problem both for animal and humans, which constitute major part of zoonoses. Some of the examples of parasitic zoonoses include cysticercosis, echinococcosis, diphylobothriasis, trichinosis, toxoplasmosis etc. [22].

Fungal zoonoses: Fungus is the main source of most of the skin problems and mostly occurs due to direct contact. Examples of fungal zoonoses include sporotrichosis [16].

\section{Socio-Economic Significance}

In 1975, the FAO/WHO expert committee on VPH classified zoonoses as follows: zoonoses that have serious effect on animal production, zoonoses that have serious consequences both for humans and for economically animals and zoonoses that have serious consequences for humans, but which are much less serious in economically important animals. this classification indicates both the health and the economic significance of zoonoses, thus stressing the fundamental importance that some of these may have upon farm management and economics. Any zoonoses that are possibly associated with occupational activities (e.g. brucellosis, bovine tuberculosis, Anthrax, Dermatomycoses, and Leptospirosis) belong to the first two categories [4].

Humans: In humans zonoses (in general occupational disease) may have various consequences including the following cost for diagnosis of the diseases, medical fees and cost of hospitalization, nursing and drugs, loss of working days or production, mortality, suffering and social consequences of disability and abandonment of farming or agricultural activities by affected or at risk persons. It should be noted that some of the above consequences are difficult to evaluate from an economic point of view and other can be mainly or exclusively evaluated in social terms. It has been observed that some zoonoses (e.g. cystic echinococcosis) may negatively affect the quality of life and that the affected patients have higher unemployment rate in comparison to the general population [22].

Livestock: in livestock the following negative consequence of zoonoses may be considered: reduced yield and quality of animal production (meat, milk, eggs, and labor), reduced birth rate and fertility, delayed performance and growth and in some cases condemnation of organ and cost for destruction of viscera and / or carcasses. There are also other possible indirect detrimental consequences, such as bans on the export of animals and their product if these are required to be free of certain infections. 
In livestock the importance of the above-mentioned economic consequences will depend to a large extent on the characteristics of the animals or on the farming or livestock industry and on the legislative rules of each country (e.g. compulsory condemnation and destruction of organ at slaughter). In some cases occupational zoonoses and related problems may exert a heavy impact on the social status of individual families and communities, especially in areas where they are highly endemic in animals, where the prevention of occupational hazards is underestimated or completely ignored and where technical assistance to farmers and stock holders and control plans are insufficient or even absent [1].

\section{Principles of Zoonoses Prevention, Control and Eradication}

There is an important distinction among the term prevention, control and eradication. Prevention is defined as inhibiting the introduction of a disease agent into an area, a specific population group or an individual. Control efforts consist of steps taken to reduce a disease problem to a tolerable level and maintain it at the level. The term control is more appropriate when a given infectious disease agent is already present. For example; an arboviral infection may be endemic in a wildlife reservoir in an area where eradication is not feasible, but its impact may be greatly reduced in people and domestic animals by vector control and immunization programs. Mastitis is impossible to completely prevent even in the best managed dairy herds, but a good control program will greatly reduce the number of clinical as well as subclinical cases [23].

Prevention and control are sometimes referred to as "primary prevention" and "secondary prevention". Primary prevention is aimed at maintaining a healthy population i.e. preventing the occurrence of a disease. Secondary prevention attempts to minimize damage after disease has occurred. Rehabilitation, after both primary and secondary prevention have failed, is sometimes referred to as "tertiary prevention", an important economic aspects of disease control and prevention programs is that, as one progresses from primary through secondary to tertiary prevention , the cost increases per unit of population [24].

Eradication is the final step in a disease control program. It consists of the elimination of a disease producing agent from a defined population or geographical area. The first serious proposal to eradicate an infectious disease (smallpox) was presented in 1767 by Dr. Maty in a paper in titled "The advantages of Early Inoculation" [25]. To achieve eradication of a disease producing agent from an area or population, it is necessary to obstruct transmission until endemicity (including carriers) ceases and prevent the reestablishment of the agent from imported sources of infection [26]. The disease may be eliminated (brucellosis from a herd of cows; tuberculosis from herds in a political subdivision) yet remains a threat to animals in that population as a result of travel of susceptible animals out of the area or import of infected animal or animal products. Brucella melitensis, for example-has not been reported from the United State goat population for more than 50 years, but people along the Mexico/ United State border have been infected with the organism. During the period 1982 through 1986,
$67 \%$ of human cases in the United State were linked to the ingestion of cheese that had been made from unpasteurized goat milk and brought across the border from Mexico [27].

There is destruction between total eradication and practical eradication. Total eradication means that the disease agent has been completely removed from the area of concern. On a worldwide base, smallpox is the only disease agent that has been totally eradicated. Practical eradication refers to the elimination of the organism from the reservoir of importance to humans or their domestic animals, rather than total eradication from the region. For example, practical eradication of canine rabies has been accomplished in the United States, whereas eradication of rabies from the wildlife reservoir has not been achieved. A frequent limitation of attempting total eradication is economic. In test and slaughter programs, for instance, the entail cost of identifying infected individual is low on a per animal basis when the prevalence is high. as the program progresses and the prevalence decreases, the effort and time required to identify the remaining infected animal increases exponentially without careful planning [28].

Practical eradication also has limitation. A primary limitation, as mentioned above with rabies, is the lack of effective methods for eradication agents from wildlife reservoirs, especially when the reservoir consists of multiple species. Attempting to eliminate vector borne agents when the vector utilizes wildlife hosts is another example of the limitation of practical eradication. It may not be difficult to remove mosquitoes in areas populated primarily by humans, but the task often becomes difficult and expensive when the ecologic niche of the vector is among wildlife. Sometimes the term "disease free" is used to describe an area in which the incidence or prevalence of a disease of interest has dropped below certain level usually an identified goal in a control program. The term was usually by the U.S. Department of Agriculture in its brucellosis eradication program. An area was designated brucellosis free when the known herd infection rate dropped below 1.0\% [28].

The basic principles of zoonoses prevention control and eradication programs are focused upon breaking the chain of transmission at its epidemiologically weakest link. Three factors are involved: the reservoir, transmission from the reservoir to the susceptible hosts, and the susceptible hosts [29]. The principle is described in the following section

Reservoir Neutralization: the ultimate source of zoonotic infection is the infected reservoir host. Whenever infection in the reservoir can be reduced or eradicated, other sources of infection progressively become less significant or disappear. Three method used to neutralize the reservoir are removing infected individual, rendering infected individuals, "non shedder" and manipulating the environment. Increasing host resistance, which will be described later as a separate control method, also can neutralize a reservoir if the number of infected animals is reduced, such as that which happened with smallpox vaccination of humans and rabies immunization of dogs. Removal of infected individual can be accomplished in two ways: test and slaughter and mass therapy [29]. 
Infection may be removed from a herd by testing and slaughtering those found to be infected. This method has been successfully used to control bovine brucellosis and tuberculosis as well as equine dourine and glanders. To be effective, a sufficiently sensitive and specific test is required, i.e. all infected animals need to be detected by the test if all infection is to be erased without removing large numbers of false-positive animals. When such a test is not available it may be necessary to use a less sensitive test, such as clinical diagnosis, on which to base a decision. Two factors that affect the decision to use test and slaughter are expense and the method of transmission. This method has been most effective with agents spread by direct transmission and in which a limited number of reservoir species are involved. The high cost to government and industry has prevented its use in many countries [26].

A second method for neutralizing the reservoir by removing infected individual is mass therapy. Mass therapy is usually restricted to a local situation in which all potentially infected animals or people are treated without first testing them to identify infected individuals. In certain situation particularly with endemic diseases in the less-developed portion of the world where diagnostic resources may be minimal, treatment without prior screening may reduce the cost factor 2 to 6 times. The cost effectiveness of mass therapy as a control method increases as the prevalence of infection in the population increases. For example, it is 10 times more cost effective if at the beginning of treatment $100 \%$ is infected rather than $10 \%$. For control purposes, the treatment must eliminate infection in carriers, not just cure clinical illness. Risks associated with mass therapy, particularly if improperly done, are the development of resistant strains of infection agents and adverse side effect [16]. The antibiotic treatment of parakeets imported into the United State, to prevent human psittacosis, and the prevention of echinococcosis by treating all dogs in a given geographic area, to break the dog-sheep cycle is example of mass therapy of animal reservoir [16].

Many of the problems associated with internal parasites of humans are being reduced by mass therapy. Diethylcarbarnazine is being used to combat fascioloiasis [28] and ivermectin is being used to combat onchocerciasis [29]. Combined with vector control, mass therapy has reduced the incidence of Schistosomiasis and African trypanosomiasis [17]. Using syndrome surveillance as a measure of success, China has reported the eradication of venereal disease (pre-AIDS) through mass therapy [30].

Environmental manipulation is a method of reservoir neutralization designed to break the chain of transmission between the portal of exit of the infected (shedder) host and the susceptible host by reducing survival of the agent in vector of vehicle (food, water, soil, vegetation). The environment of concern is wherever the agent may be found outside the vertebrate host. A limitation is that it is a local measure, effective only in the immediate area where the control is instituted. Various parasitic control strategies that provide example of this approach are proper fecal waste disposal (acting on the portal of exit), disinfection of fecal wastes, and pasture rotation to decrease exposure of susceptible hosts (portal of entry) [29].
Provision of adequate toilet facilities, coupled with education and supervision to ensure their use, will prevent the spread of Taenia saginata from feedlot employees to cattle. If facilities are not convenient, employees may use haystacks, feed bunks, or other location, which can result in contamination of cattle feed [28]. The use of fermentation lagoons to destroy agents transmitted by the fecal-oral route has proven very successfully. Recently compositing using aerobic, thermophilic bacteria has been introduced as a costeffective method for reducing the viability of infective organisms contained in organic wastes. If human wastes are to be used for fertilization of pastures, it is important to first provide proper sewage treatment to prevent transmission of viable parasites ova before the effluent is applied to pastures [31].

Introduction of sterilized males, which has been very effective in eradicating screwworms, depends upon the life cycle and breeding patterns of the arthropod. This method would not be as effective in controlling screwworm if the proportion of non-irradiated males was higher or if the female flies bred with more than one male. Biological control using natural predator or pathogens of vectors has been used with some success to reduce mosquito population. Examples are introduction of Gambusia fish to consume mosquito larvae and use of a competitor snail, Marisa cornuarietis, to drive out Australorbis spp. snails, an intermediate host of Schistosomes. This technique, however, is dependent upon the population density of the vector and has ecologic limitation [31].

Reducing Contact Potential: A basic principle in preventing direct transmission of an infectious agent from an infected individual to a susceptible host is to reduce the opportunity for contact. In disease control two population are considered, the known infected and the potentially exposed susceptible. Three methods are used: isolation and treatment of cases, quarantine of possibly infected individuals, and population control. Note the different use of terms, isolation of known cases of infection and quarantine of individuals suspected of having been exposed. Simply put, isolation is designed to keep the agent in, whereas quarantine is designed to keep the agent out. The laminar flow hood, familiar to all who have worked in a microbiology laboratory is an application of the basic principle of isolation, keeping the agent in (the hood), thereby protecting the technician. Herd immunity which will be described under the topic of disease control by increasing host resistance, also is a method for reducing contact potential. When the proportion of immune animals in a given population is sufficiently great, a disease agent transmitted by direct contact cannot enter and spread because the opportunity for contact between infected (shedder) animals and susceptible animals has been reduced [16].

Isolation of an infected clinically ill animal has two advantage; it reduces the probability of contact with a susceptible lost and facilitates treatment and disinfection. Because this approach depends on early and accurate diagnosis from effective disease control programs based on isolating and treating cases to prevent the spread of infectious disease agent have serious shortcoming and frequently fail. Furthermore, the approach is generally in effective if shedding occurs during the incubation period or if healthy carriers are shedding. An exception is when all animals in a group are 
isolated as, for example when individual calving facilities are used in Brucella reactors herd [16].

Quarantine of apparently healthy individuals that may have been exposed to a source of infection had its beginning during the second plague pandemic. When ships arrived from the Middle East where plague was endemic, the crews were restricted from landing for 40 days after arrival. By experience, the authorities know that this was how long to wait to see if any human cases occurred. It was also how long it took for infected fleas to die. To be effective, quarantine must be enforced for the longest incubation period of the disease in question, to see if the suspected infected individuals developed detectable disease. It is not effective with disease involving chronically infected healthy shedders. The population quarantined may be a single animal as with a biting dog suspected of being rabid or may include an entire herd of cows exposed to tuberculosis to see if they become tuberculin positive. Geographic area that is free of rabies such as the United Kingdom and Hawaii, utilize 4 to 6 months quarantine for dogs and cats.

The "closed herd" concept is an application of quarantine. A farmer operating a dairy herd under this principle will raise all replacement stock rather than purchasing replacement and by so doing; reduced the risk of introducing an agent of disease. This procedure demands an excellent reproduction program but is advantageous because the need for protective immunization reduced to the bare minimum. Portable pens for raising dairy calves allow some quarantine space between calves to control agents spread by direct contact. Their portability is especially useful in controlling gastrointestinal parasitism, for the pens can be moved regularly to facilitates cleaning the ground to break the cycle of transmission. The placement of animals whether in laboratory animal colonies, farms or feedlots, should be done with the principles of isolation and quarantine in mind, establishing flow patterns that minimize contact between healthy susceptible animal and sources of infection. Animals, and animal products, feedstuffs, bedding, vehicles, water sources and personnel must all be evaluated as potential sources of disease agents [16].

Population control program are other methods of reducing contact. Control may be relatively benevolent, as is seen with leash law and with the restriction of movement of sheep through human population canter in towns in the Western United State during lambing season (when $\mathrm{Q}$ fever occurs from airborne spread). On the other hand, population control may be very drastic, as when population reduction is utilized. Leash laws have been designed primarily to control rabies and reduced fecal contamination. Dogs, defecating on beaches and parks where there are children sandboxes, deposit ascarid ova, which if ingested can produce visceral larvae migrans (VLM) and hookworm larvae which can penetrate the skin resulting in coetaneous larva migrans (CLM). CLM and VLM, as well as toxoplasmosis, are also problems related to cats as a source of soil contamination, but movement of cats is even more difficult to control.

Leash laws are theoretically appealing but have never been very effective because of public apathy -if not resistance and lack of enforcement. This resistance to leash law was true in 1887, when rabies was made a notifiable disease in London and is true today [17]. A survey conducted in 1975 discovered that, although $95 \%$ of U.S cities had leash laws, only $52 \%$ reported that they were effective in reducing strays. In a crisis, however public awareness and cooperation will increased and may even permit more effective procedures, such as large-scale vaccination programs [31].

Increasing Host Resistance: in addition to neutralization of reservoirs or contact reduction, zoonoses may also be controlled by increasing host resistance to infection. Preventing infection is the ideal, but in many instances, increasing host resistance may only lessen the severity of disease, without an equal increase in resistance to infection [29].

In veterinary medicine, genetic selection for resistance and reducing stress by improving nutrition or by better shelter is routine procedure. In Africa, the N'Dama and West African short horn cattle breeds, which have been resident there for 5,0007,000 years are favored because of their trypano-tolerance. In the southern United State, range beef cattle of European breeds are frequently bred to include a $25 \%$ Brahma parentage because of the latte's greater resistance it tick infestation.

Maintaining animals at a proper level of nutrition not only increase their ability to resist infection but also increases their ability to respond properly to immunization. In human medicine genetic selection for resistance may occur naturally, as with sickle cell anemia and resistance to malaria, but is not acceptable as an applied disease control procedure. Reduction of stress, by providing improved shelter and nutrition is not only an end but is also a means to reduce the ravages of epidemics by increasing the survival ability of the affected population. This is well recognized with greater case fatality rates among starving population during epidemics. These approaches to disease control will not be explored further in this text. There are however, two procedures for increasing host resistance which is appropriate for presentation; chemoprophylaxis and immunization [30].

Chemoprophylaxis: contrasts with mass therapy in that, in the latter, medication is administered on the assumption that the recipient is infected whereas chemoprophylaxis attempts to prevent infection or at least reduced the severity of the disease. In contrast to immunization it is passive means of increasing host resistance, lasting only if the drug lasts. Active response to immunization, however, lasts for months or even a lifetime. Typically, no host response is elicited. Live anthrax vaccine with concurrent penicillin prophylaxis is an example of chemoprophylaxis of the wrong kind, i.e. no infection and no immunity [31].

Chemoprophylaxis is used in laboratories when personnel are accidentally exposed to an agent (including several zoonotic) known to be susceptible to a drug. This is to prevent infection which differs from mass therapy, which is used to eliminate infection. On the other hand, mass therapy may leave some immunity in those who were previously infected. Chemoprophylaxis may involve adverse reaction to the drug. in some instance the agent may be resistant [14]. 
Typically, chemoprophylaxis is applied when no other more effective means of protecting the host is available. Anti-malarial medication for people is an example. The medication does not prevent infection (injection of sporozoites by the mosquito) but reduced the severity of consequences of malaria by suppressing the erythrocytic stage. Malaria drug are no longer used in those areas of the world where the disease has been eradicated. Chemoprophylaxis is a consideration for any high-risk groups when an effective drug is available and suitable immunization or adequate protective clothing is not [13].

In veterinary medicine slow or pulse-release boluses of oxfendazole have been demonstrated to be effective in reducing parasitic gastroenteritis (and pasture contamination) among bovines. Some of the most widely used chemoprophylactic materials for domestic animals are insect repellents to ward off arthropod vectors and anti-heart worm medication for dogs. Both procedures are to edged swords, for them also present risks. Cats are easily made ill by exposure to certain chemical repellents. A prophylactic dose of an anti-heartworm medication given to a dog already infected with the parasite can cause death, because adult worms are killed and dislodged from their site in the heart, producing emboli [31].

Immunization: vaccine is used for two purposes, to protect susceptible individual from infection or disease and to prevent transmission of infectious agents by creating an immune population. To be most effective in controlling disease, the stimulus of immunization should be sufficient to prevent infection as well as disease. In maintenance hosts, no reduction in the reservoir of infection occurs if only disease is prevented. The risk of disease remains from any susceptible individuals introduced into the population if carriers persist. The level of immunity needed to prevent disease is not necessarily the same as the level needed to prevent infection. The efficiency of immunization as a method for disease control is measured in terms of the percentage of the population developing the desired level of protection in relation to the resources expended (vaccine, equipment, labor, promotion etc.). Efficiency increases as the number of doses needed decreases. Assuming a similar percentage of the population reaches a level of protection enough for control by either method, vaccine in the drinking water would be far more efficient than administration by injection [29].

In planning any immunization program, the first step is to identify the population at risk (susceptible likely to be exposed) and then to decide on the specific disease control goal, e.g. reducing the incidence of disease to just a few sporadic cases elimination of the agent. The decision to vaccinate or not to vaccinate is based on relative risk. When choosing which route to take in relation to each disease, ask the question where, when, who, and why? The answer to one or more of these questions may be crucial to determine the correct decision [30].

Immunization as a method of disease control is generally so effective and commonplace that all too often, the many variables associated with the procedures are ignored [14]. Immunization failures may occur as the result of failure of the delivery system or failure of the immune response, or they may be iatrogenic in origin [31].

\section{Conclusion and Recommendations}

Zoonoses are infections naturally transmitted between vertebrate animals and humans. Zoonoses may be bacterial, viral or parasitic or may involve un-conventional agents. The major routes of transmission of zoonotic disease are direct or indirect contact, aerosol, and vector borne. Zoonotic disease depends upon three points i.e. nature of etiologic agent, reservoir host and life cycle of the infecting organism, major impact of zoonoses include illness, monetary loss, adverse effect on morale of personnel, unfavorable publicity and medico-legal implication. The basic principles of zoonoses prevention, control and eradication involves reservoir neutralization, reducing contact potential and increasing host resistance.

Based on the above conclusion the following recommendations are forwarded

1. Network among public health, medical, veterinary laboratories and research Centre working with zoonotic pathogens should be established or strengthened.

2. Health professionals should educate the public about zoonotic diseases to effectively practice prevention and control strategies.

3. Reservoir neutralization, reducing contact potential and increasing host resistance must be done to prevent, control and eradicate zoonotic diseases.

\section{References}

1. WHO (2010) Managing zoonotic public health risks at the human animal ecosystem interface Strong intra-sectoral partnerships in health, food safety and zoonoses?

2. WHO (2005) The control of Neglected Zoonotic Diseases Report of a joint WHO/DFIDAHP Meeting with the participation of FAO and OIE? Geneva, Switzerland.

3. Nkuchia M, Ruth L, Chris A, Henriette V (2007) Infectious diseases surveillance. Blackwell publishing Inc USA pp 246-248.

4. WHO (2004) Water borne Zoonoses, Identification, Causes and Control.

5. Jonathan R, Joshua L (2006) Emerging infectious diseases from the global to the local prospective. A summary of a workshop of the forum on Emerging infections. National Academy Press Washington, USA.

6. Meslin F, Stohr K, Heymann D (2000) Public health implications of emerging zoonoses. Rev Sci Tech 19(1): 310-317.

7. Smits H, Cutler S (2004) Contributions of biotechnology to the control and prevention of brucellosis in Africa. Afr J Biotechnol 3(12): 631-636.

8. McDermott J, Arimi S (2002) Brucellosisin sub-Saharan Africa: epidemiology control and impact. Vet Microbiol 90(1-4): 11-134.

9. Yamada A (2004) Zoonoses. Uirusu 54: 17-22.

10. OIE (2010) Terrestrial Animal Health Code.

11. Cleaveland S, Laurenson M, Taylor L (2001) Diseases of humans and their domestic mammals: Pathogen characteristics, host range and the risk of emergency. Philos. Trans. Roy Soc Lond B Biol Sci 356 (1411): 991-999. 
12. WHO/FAO/OIE (2004) Report of the WHO/FAO/OIE joint consultation on emerging zoonotic diseases. Geneva, Switzerland 3-5.

13. Bereket $T$ (2008) Prevalence and economic impact of bovine hydatidosis at AA abattoir, DVM Thesis, FVM, DZ, Ethiopia. P. 12.

14. Taylor MA, Coop Rl, Wall RL (2007) Veterinary Parasitology. ( $3^{\text {rd }}$ edn), Blackwell publishing Iowa, USA pp. 337-339.

15. Lenhart S, Seitz T, Trout D, Nancy Bollinger (2004) Issues affecting respiratory selection for workers exposed to infectious aerosols: emphasis on health care settings. Appl Biosafety 9: 20-36.

16. Acha P, Szyfres B (2003) Zoonoses and communicable disease common to man and animals Volume 2: chlamydioses, rickettsioses and viruses. ( $3^{\text {rd }}$ edn), Pan American Health Organization, Washington, USA.

17. McQuiston J, Childs J (2002) Q Fever in human and animals in the United State, Vector Borne Zoonotic Dis 2: 179-191.

18. Reddy M (2007) Bird flu. Health, Safety and Contingency Guidelines. SBS Publishers and Distributors New Delhi.

19. Wong K, Shieh W, Zaki S, Tan C (2002) Nipah virus infection, an emerging paramyxoviral zoonosis. Spring Semin mmunopathol 24(2): 215-228.

20. Richmond J, Krauss H, Weber A, Appel M, Enders B, et al. (2003) Zoonoses, Infectious Diseases Transmissible from Animal to Humans $\left(3^{\text {rd }}\right.$ edn). ASM Press. American Society for Microbiology, Washington DC, USA.

21. Leroy E, Kumulungui B, Pourrut X, Rouquet P, Hassanin A, et al. (2005) Fruit bats as reservoirs of Ebola virus. Nature 438(7068): 575-576.

22. Torgerson P, Karaeva R, Corkeri N, Abdyjaparov T, Kuttubaev O, et al (2003) Human cystic echinococcosis in kyrgystan: an epidemiological study. Acta trop 85(1): 51-61.
23. Henttonen H, Fuglei E, Gower N, Haukisalmi V, et al. (2001) Echinococcus multilocularis on Svalbard: introduction of an intermediate host has enabled the local lifecycle. Parasitology 123(6): 547-552.

24. Sohn H, Probert W, Glaser C, Gupta N, Bollen W, et al. (2003) Human neurobrucellosis with intracerebral granuloma caused by a marine mammal Brucella spp. Emerg Infect Dis 9(4): 485-488.

25. Cockburn T (1967) The evolution of human infectious diseases infectious disease their evolution and eradication. Spring field IL Charles C Thomas pp 84-107.

26. Schellenberg R, Tan B, Irvine J, Stockdale R, Gajadhar A, et al. (2003) An outbreak of trichinellids due to consumption of bear meat infected with Trichinella nativa, in 2 northern Saskatchewan communities. J Infect Dis 188(6): 835-843.

27. Harvey RA, Champe PC, Fisher BD (2007) Lippincott's Illustrated Reviews: Microbiology. ( $2^{\text {nd }}$ edn), Lippincott Williams, Wilkins, Philadelphia pp. 227-228.

28. Ibrahim MM (2010) Study of cystic echinococcosis in slaughtered animals in $\mathrm{Al}$ Baha region, SaudiArabia: interaction between some biotic and abiotic factors. Acta Tropica, 113(1): 26-33.

29. Martinma EA (2007) Oxford Concise Medical Dictionary. ( $7^{\text {th }}$ Edn), Oxford University Press, Bungar, UK pp. 342-343.

30. Beers MA, Porter RS, Jones TV, Kaplan JL, Berkwits M (2006) The Merck Bailleir Tindall, London, UK, pp. 809.

31. Jones TC, Hunt RC, King NW (2006) Veterinary Pathology. ( $6^{\text {th }}$ Edn), Blackwell Publishing, USA, pp. 655-656. 